\title{
Low-temperature magnetic behavior of the organic-based magnet $\mathrm{Na}\left[\mathrm{FeO}_{6}\left(\mathrm{C}_{10} \mathrm{H}_{6} \mathrm{~N}\right)_{3}\right]$
}

\author{
V.P. Dyakonov ${ }^{1,2}$, E. Zubov ${ }^{1}$, E. Aksimentyeva ${ }^{2}$, K. Dyakonov ${ }^{4}$, \\ S. Piechota ${ }^{3}$, and H. Szymczak ${ }^{3}$ \\ ${ }^{I}$ A.A. Galkin Donetsk Physico-Technical Institute, NASU, 72 R. Luxembourg Str., Donetsk 83114, Ukraine \\ E-mail: zubov@fti.dn.ua \\ ${ }^{2}$ Lviv Ivan Franko National University, 6 Kirila-Mefodia, Lviv 79005, Ukraine \\ ${ }^{3}$ Institute of Physics, PAS, 02-68 Warszawa, Al. Lotników 32/46, Poland \\ ${ }^{4}$ A.F. Ioffe Physico-Technical Institute RAN, 26 Politekhnicheskaja Str., St.-Petersburg 194021, Russia
}

Received February 3, 2014, published online May 21, 2014

\begin{abstract}
The static and dynamic aspects of magnetic behavior of the complex of iron with 1-nitroso-2-naphthol $\mathrm{Na}\left[\mathrm{FeO}_{6}\left(\mathrm{C}_{10} \mathrm{H}_{6} \mathrm{~N}\right)_{3}\right]$ as a function of temperature, frequency and magnetic field have been analysed. The ac susceptibility $\left(\chi_{a c}\right)$ and dc magnetization $\left(M_{d c}\right)$ were measured over a temperature range of 1.5-200 K in external magnetic field up to $90 \mathrm{kOe}$ and over a frequency range of $95-2000 \mathrm{~Hz}$. The experimental data indicate the absence of magnetic long range order in this complex. The magnetization does not reach a saturation in field of $90 \mathrm{kOe}$ at $1.5 \mathrm{~K}$. At low temperatures, the following characteristic peculiarities of magnetic behavior of complex studied have been found, namely : cusp-like anomalies in the ac susceptibility and zero field coold (ZFC) magnetization at $T_{\text {cusp }}=$ $=17 \mathrm{~K}$; frequency dependence of the $T_{\text {cusp }}$ temperature; remanence and time-dependent relaxation of ZFC magnetization. Attempts were made to compare the $\chi_{a c}$ and $M_{d c}$ anomalies in complex studied with those of other magnetic systems. Comparison of characteristic peculiarities of magnetic behavior of the spin-glasses and superparamagnets was also performed. Although superparamagnetic behavior should not be completely ruled out as an explanation of the freezing phenomena in sample studied, the analysis of experimental results strongly suggests that the spin-glasslike behavior is a more consistent explanation.
\end{abstract}

PACS: $75.10 . \mathrm{Nr}$ Spin-glass and other random models;

75.20.Ck Nonmetals;

75.60.Ej Magnetization curves, hysteresis, Barkhausen and related effects;

75.50.Lk Spin glasses and other random magnets.

Keywords: magnetization, susceptibility, spin glass, superparamagnet.

\section{Introduction}

The magnetism of the magnetic materials were always associated with the unpaired electrons located on metal atoms or ions. Today, many examples of organic- and molecule-based materials are discovered which exhibit magnetic ordering including ferromagnets, ferrimagnets, antiferromagnets, spin-canted structures and spin glasses. The problem of magnetism in organic materials is one of the important in physics of low-dimensional systems. The interest in molecular magnets is twofold, from both the basic and applied research perspectives. Organic-based magnets attract attention due to the ability to chemically tune their magnetic properties. They might be able to replace in some applications typical magnetic materials and to represent unique experimental realizations of theoretical models. One of the first examples of molecular magnets are the structures with intrinsic uncompensated spins (organic free radical-based magnets). Interactions between the unpaired electrons in organic free radical-based magnets generally lead to antiferromagnetic (AFM) exchange. The highest temperature of canted antiferromagnetic ordering $\left(T_{N}=\right.$ $=36 \mathrm{~K}$ ) for purely organic free radical has been reported for p-NC. $\mathrm{C}_{6} \mathrm{~F}_{4} \cdot \mathrm{CNSSN}$ [1]. The Curie temperatures of ferromagnetic (FM) materials consisting of purely organic radical are very low (for example, $T_{C}=1.48 \mathrm{~K}$ is found for Rassat's radical [2]). 
An alternative approach to organic magnets may be based on $\pi$-conjugated polymers with large magnetic moment and magnetic order at low temperatures [3-6].

In parallel activity, the extensive studies of organometallic magnets with the aim to find systems with high magnetic ordering temperature have been carried out. Some of the them have the Curie temperature close to room temperature [7-12 and references therein].

Investigations of the static and dynamic magnetic behavior of the organic-based magnets help to study an influence of their structure on macroscopic magnetic properties as well as to elucidate the mechanisms of molecular magnetism. In series of works [13-16], the observation of magnetic behavior in an organic conjugated polymers resembling those of spin glasses (SG) or superparamagnets (SP) below the blocking temperature was reported.

In this paper, the studies of the magnetic properties of the complex of iron with 1-nitroso-2-naphthol $\mathrm{C}_{10} \mathrm{H}_{7} \mathrm{NO}_{2}$ in the form of sodium salt $\mathrm{Na}\left[\mathrm{FeO}_{6}\left(\mathrm{C}_{10} \mathrm{H}_{6} \mathrm{~N}\right)_{3}\right]$ are presented. The investigations have been performed over a temperature range of 1.5-200 K, in external magnetic fields up to $90 \mathrm{kOe}$ and over a frequency range of 95-2000 Hz. The polymer complex studied was found to exhibit a spin-glass-like features of dc magnetization and ac susceptibility. This fact was proved by comparison of the macroscopic and intrinsic properties of this complex with properties of spin glass systems, which were investigated earlier. The reliability of our interpretation was confirmed by performing several experiments of different kind on the same sample.

\section{Experimental}

The sodium salt of 1-nitroso-2-naphthol iron complex, $\mathrm{Na}\left[\mathrm{FeO}_{6}\left(\mathrm{C}_{10} \mathrm{H}_{6} \mathrm{~N}\right)_{3}\right]$, was synthesized by the reaction of bisulfite derivative of 1-nitroso-2-naphthol and iron (II) sulfate at the presence of $\mathrm{NaOH}$ and $\mathrm{Na}_{2} \mathrm{CO}_{3}$ according to a procedure described in Ref. 17. Molecular structure of the $\mathrm{Na}\left[\mathrm{FeO}_{6}\left(\mathrm{C}_{10} \mathrm{H}_{6} \mathrm{~N}\right)_{3}\right]$ complex is shown in Fig. 1 .

In this complex, the $\mathrm{Fe}$ ion is surrounded by six oxygen atoms belonging to three molecules of an organic ligand

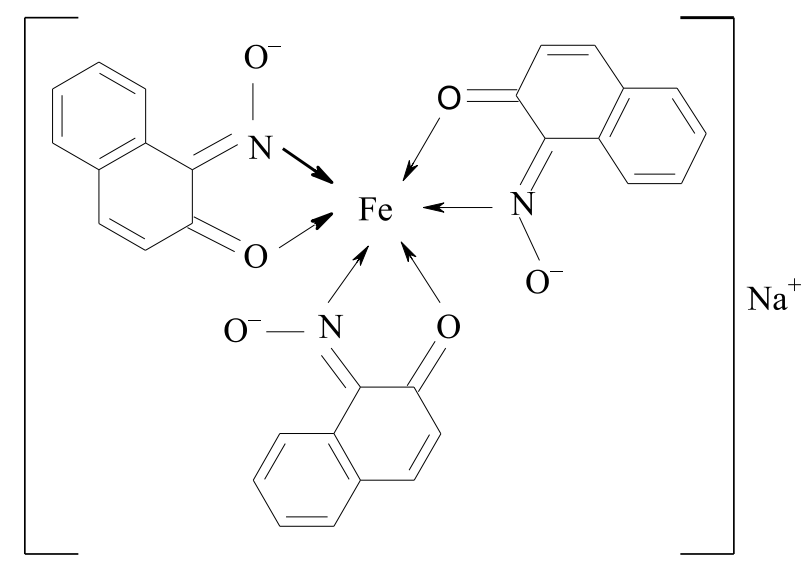

Fig. 1. Molecular structure of the $\mathrm{Na}\left[\mathrm{FeO}_{6}\left(\mathrm{C}_{10} \mathrm{H}_{6} \mathrm{~N}\right)_{3}\right]$ complex.
$\mathrm{Na}\left[\mathrm{FeO}_{6}\left(\mathrm{C}_{10} \mathrm{H}_{6} \mathrm{~N}\right)_{3}\right]$ [17]. According to the $\mathrm{x}$-ray powder diffraction measurements performed at room temperature ( $\mathrm{CuK}_{\alpha}$-radiation), the iron-1-nitroso-2-naphthol complex is the compound with a low crystallographic symmetry. Its crystal structure was determined to be monoclinic with the spatial group $P 2{ }_{1}$. The lattice parameters are: $a=11.157 \AA$, $b=8.76 \AA$, and $c=6.203 \AA$. The monoclinic angle is equal to $\beta=92.40^{\circ}$. Magnetic properties of this complex are mostly determined by the presence of Fe ions subjected to the influence of the crystal field created by the molecules of organic ligands.

In this paper, the experiments of different kind being important for understanding of the magnetic state of $\mathrm{Na}\left[\mathrm{FeO}_{6}\left(\mathrm{C}_{10} \mathrm{H}_{6} \mathrm{~N}\right)_{3}\right]$ complex have been performed. Magnetization, $M(T, H)$, measurements were carried out using a vibrating sample magnetometer over a temperature range of 5-200 K. The zero-field-cooled (ZFC) and field-cooled (FC) magnetization were measured over a magnetic field range of $0.1-16 \mathrm{kOe}$. Real $\left(\chi^{\prime}\right)$ and imaginary $\left(\chi^{\prime \prime}\right)$ parts of the ac susceptibility $\left(\chi_{a c}\right)$ were measured using a Lake Shore 7229 ac susceptometer with an excitation field $h_{a c}=$ $=5.0$ Oe over a temperature range of $1.5-140 \mathrm{~K}$ and in a magnetic field up to $5 \mathrm{kOe}$. The temperature dependence of $\chi_{a c}$ at different frequencies was measured with heating the sample in zero field. The frequency was varied in the range of $95 \mathrm{~Hz} \leq f \leq 2000 \mathrm{~Hz}$. The magnetization isotherms (at $T=1.5$ and $T=5 \mathrm{~K}$ ) were measured in magnetic field up to $90 \mathrm{kOe}$. The magnetic measurements were carried out on pressed powder pellets of cylindrical shape $3 \mathrm{~mm}$ in diameter and $6 \mathrm{~mm}$ in length.

\section{Results and discussion}

The temperature dependence of in-phase component of ac magnetic susceptibility, $\chi^{\prime}(T)$, shows a steep rise below $50 \mathrm{~K}$, with a cusp visible at $T_{f} \approx 17 \mathrm{~K}$ (Fig. 2(a)). The $\chi^{\prime}(T)$ dependence above $T_{f}$ was fitted by the CurieWeiss law of $\chi=\chi_{0}+C /(T-\theta)$ with $\chi_{0}=0.0017 \mathrm{emu} / \mathrm{mol}$, the Curie constant $C=0.46 \mathrm{emu} \cdot \mathrm{K} / \mathrm{mol}$ and the Weiss constant $\theta_{A F M}=-2.4 \mathrm{~K}$. The small $\theta_{A F M}$ value indicates a relatively small exchange, and the negative sign of $\theta_{A F M}$ indicates a predominant antiferromagnetic character of exchange interactions.

It is well known that the cusp-like anomaly is observed in traditional SG systems [18]. The susceptibility of the usual Heisenberg antiferromagnet displaying a long-range ordering also shows a sharp peak at the Nèel temperature. In order to clarify both the characteristic features and the origin of occurrence of the $\chi^{\prime}(T)$ maximum, we have measured a variation of the temperature dependence of ac susceptibility with a change of both ac field frequency and dc magnetic field strength.

Typical examples of temperature measurements performed in the field of $H_{d c}=0$ for different frequencies $f=$ $=95,500,1000$, and $2000 \mathrm{~Hz}$ are depicted in Figs. 2 (a),(b). 

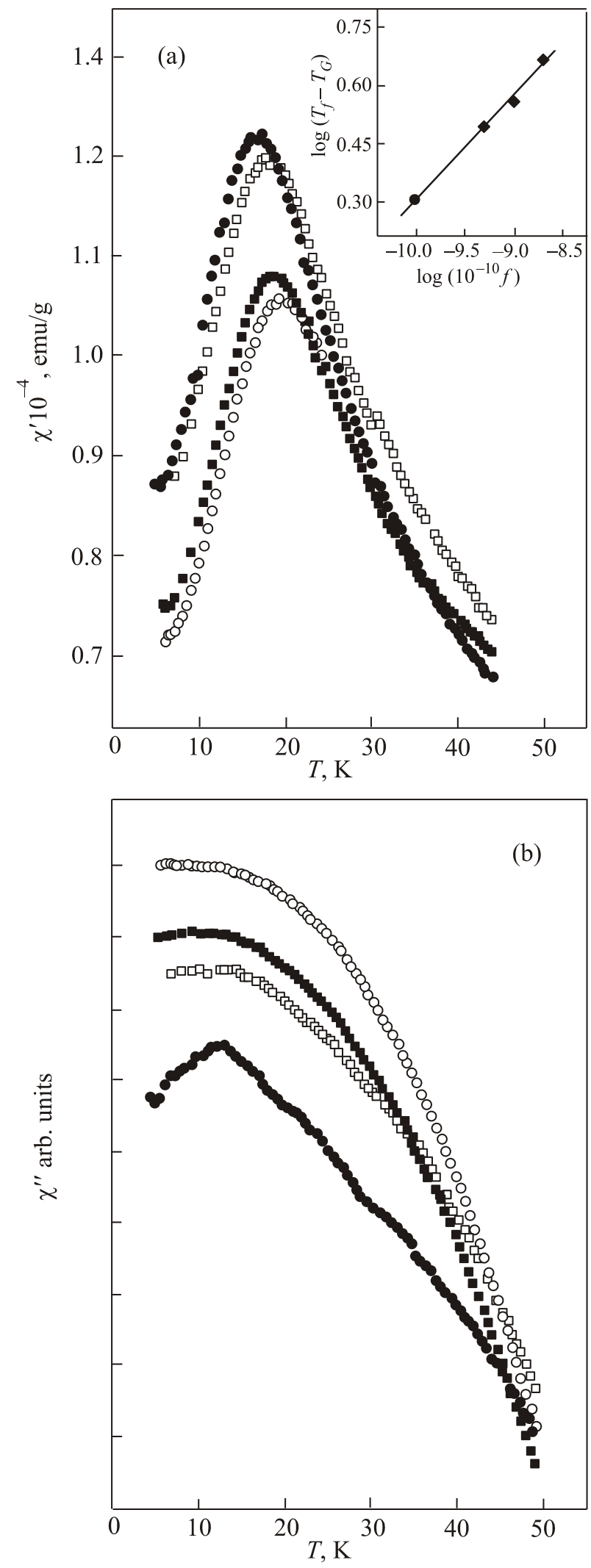

Fig. 2. Temperature dependences of $\chi^{\prime}$ (a) and $\chi^{\prime \prime}$ (b) components of the ac magnetic susceptibility at different frequencies $f, \mathrm{~Hz}: 95$ $(\bullet), 500(\square), 1000(\square), 2000(O)$. In Fig. 2(b), the vertical axis is shifted at each frequency. Inset to Fig. 2(a) shows the log-log plot of the frequency dependence of $\left(T_{f}-T_{G}\right)$.
The $\chi^{\prime}(T)$ anomaly (Fig. 2(a)) is accompanied by the onset of an out-of-phase component of ac susceptibility, $\chi^{\prime \prime}$, near $T_{f}$ (Fig. 2(b)). As it is seen in Fig. 2(a), the $\chi^{\prime}$ versus $T$ curves display a frequency-dependent peak at $T_{f}$ which shifts towards higher temperatures as a frequency increases. With increasing frequency, the peak of the $\chi^{\prime}(T)$ dependence decreases and the maximum observed on the $\chi^{\prime \prime}(T)$ curve becomes progressively rounded and less intense.

A presence of the frequency-dependent maximum on the $\chi^{\prime}(T)$ dependence is often taken as a sign of either spin glass or superparamagnetic behavior. To distinguish between these cases, we have estimated the value of the relative variation of the peak $T_{f}$ temperature per decade of frequency $-K=\left(\Delta T_{f} / T_{f}\right) /[\Delta \log (f)]$ using the frequency dependence of $T_{f}$. Here $\Delta$ refers to differences between measurements at different frequencies. The calculated $K$ value is equal to 0.09 , which is close to the extreme limit of 0.08 for traditional spin glasses, while for SP the $K$ value is greater than 0.1 [18]. Thus it should be noted that the $\chi^{\prime}(T)$ susceptibility of the studied complex has a cusp-like anomaly at $T_{f}$ observed in SG, while for SP the $\chi^{\prime}(T)$ curve shows a broad maximum and is slightly frequencydependent above $T_{f}$. The position of a cusp on the $\chi^{\prime}(T)$ dependence as a function of frequency was used to define a spin-glass freezing temperature.

Another property of spin glasses is the dynamic slowing down of the fluctuations near the spin glass transition temperature which has the well-known power law dependence

$$
T_{f}=T_{G}\left[1+\left(\tau_{0} f\right)^{1 / z v}\right]
$$

where $T_{G}$ is the critical temperature of phase transition to spin glass state at zero frequency, $z v$ is the dynamic critical exponent and the $\tau_{0}$ value is usually taken equal to $10^{-13} \mathrm{~s}$ [18]. If one plots the measuring $f$ frequency as a function of $\left(T_{f}-T_{G}\right)$ difference in a log-log plot, as shown in inset to Fig. 2(a), then in the result of fitting by a straight line one obtains the $z v$ value of about 4 . This dynamic exponent is close to the minimal value characteristic for spin glasses [18].

The susceptibility decreases and the cusp in the $\chi(T, H)$ curves becomes more rounded with increasing $H_{d c}$ field (Figs. 3 (a),(b)). In magnetic field of 1000 Oe, the anomaly in the $\chi(T)$ dependences disappears completely. The behavior of ac susceptibility as a function of both frequency and magnetic field is similar to that which is observed for ordinary SG systems. However, the $\chi^{\prime}(T)$ curves measured in different dc fields do not tend to intersect at low temperatures, in contrast to the case of SG systems. With decreasing temperature, the $\chi^{\prime}$ susceptibility tends to flatten out and then turns upwards (Figs. 2(a) and 3(a)). This behavior can indicate the existence of the isolated Fe spins showing a Curie-like behavior at low temperatures. 

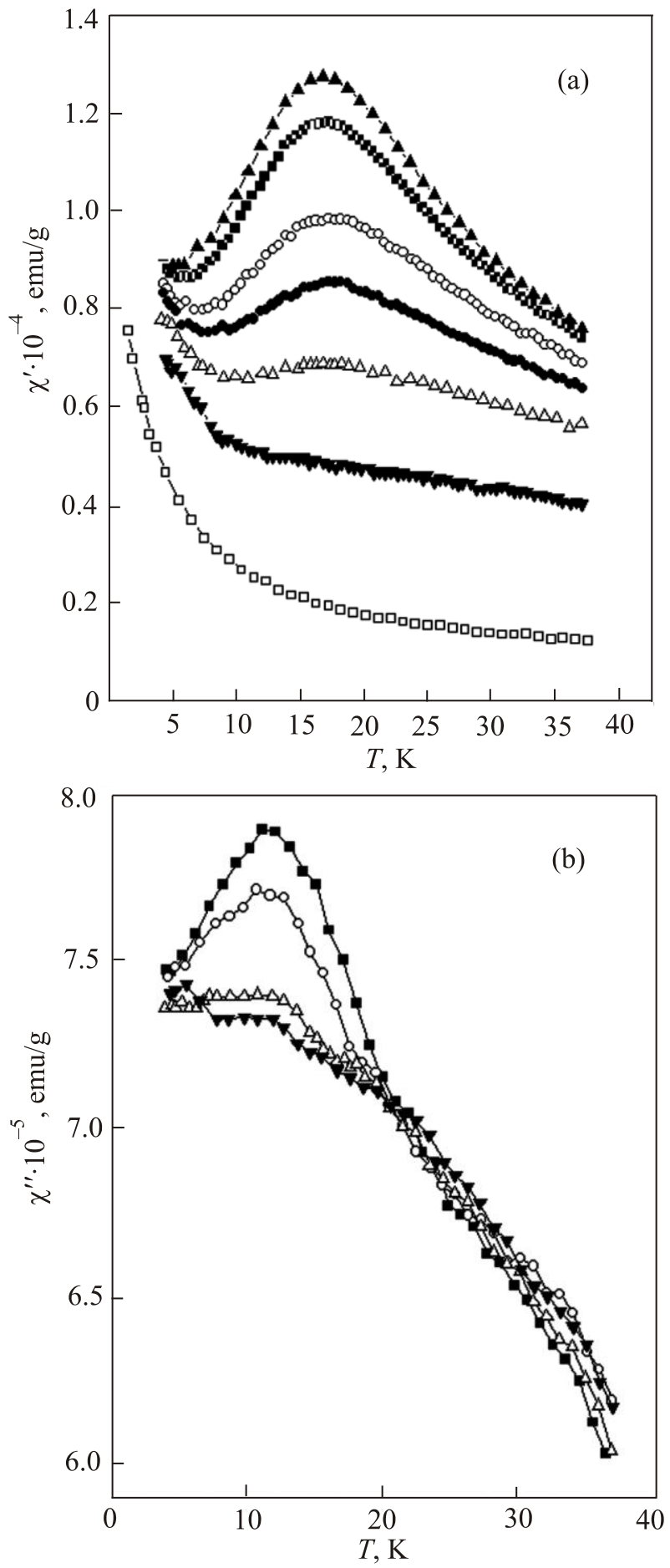

Fig. 3. Temperature dependences of $\chi^{\prime}$ (a) and $\chi^{\prime \prime}$ (b) components of the ac magnetic susceptibility at frequency of $95 \mathrm{~Hz}$ for different fields $H$, Oe: $0(\bullet), 100(\bullet), 200(\bigcirc), 300(\bullet), 500(\Delta), 1000$ $(\nabla)$, and $5000(\square)$.

The temperature dependence of magnetization, $M(T)$, was studied at two regimes of the sample cooling: zero field cooling and field cooling. As it will been shown below, the cooling conditions influence on a magnetic behavior of complex studied. The ZFC magnetization measured as a function of temperature in a constant magnetic field at heating regime displays a maximum (Fig. 4). The $M_{Z F C}(T)$

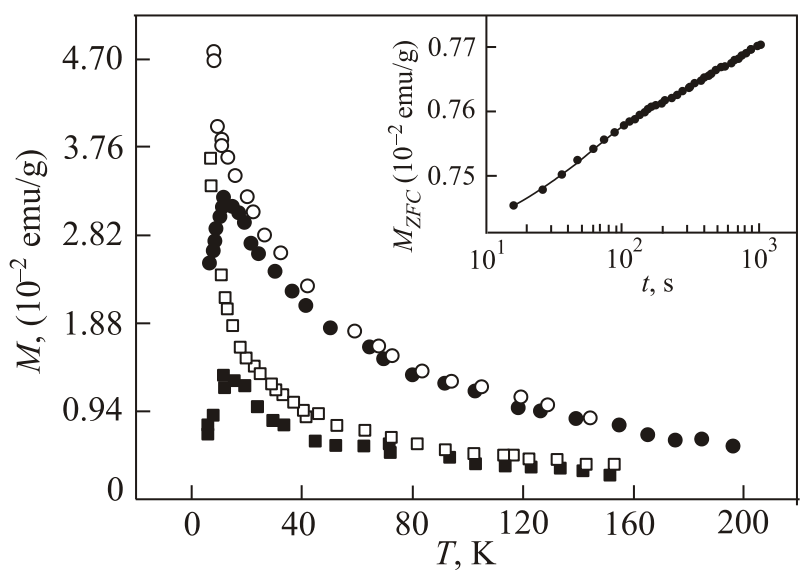

Fig. 4. Zero-field-cooled $(\mathbf{\square} ; \bullet)$ and field-cooled $(\square, \bigcirc)$ magnetization for fields of $H=0.1$ and $0.5 \mathrm{kOe}$, respectively. Inset shows the time evolution of $M_{Z F C}$ magnetization at 100 Oe after zero-field cooling from room temperature to $5 \mathrm{~K}$, for waiting time $t_{w}=100 \mathrm{~s}$.

behavior is similar to the $\chi^{\prime}(T)$ dependence. The FC magnetization increases with decreasing temperature below $T_{f}$ and shows no maximum in contrast to the behavior of conventional spin glasses.

The temperature, where $M_{F C}(T)$ and $M_{Z F C}(T)$ curves split, can be used to define the irreversibility temperature $\left(T_{\text {ir }}\right)$. A difference between $\mathrm{ZFC}$ and FC magnetization observed below $T_{\text {ir }}$ indicates the freezing of the magnetic moments. The irreversibility increases as the temperature decreases and becomes more pronounced near $T_{f}$. A behavior of both FC magnetization and irreversibility with decreasing temperature are characteristic of anisotropic materials. It should be noted that the $T_{\mathrm{ir}}$ temperature is quite high, in contrast with both the typical SG systems, where the irreversibility starts below $T_{f}$, and the SP, where it occurs just at $T \approx T_{f}$. The difference between $M_{F C}$ and $M_{Z F C}$ is much greater in the sample studied than in the SG systems at low temperatures. As it is shown below, the divergence of the FC and ZFC magnetization below the $T_{\text {ir }}$ temperature is associated with both the opening up of the hysteresis loop and the definite value of coercitivity, which are a measure of the magnetocrystalline anisotropy. The $T_{\mathrm{ir}}$ temperature depends on a applied magnetic field, namely, shifts towards lower temperatures with increasing $H_{d c}$. As $H_{d c}$ increases to about $5 \mathrm{kOe}$, the $T_{\text {ir }}$ and $T_{f}$ temperatures coincide.

Both the cusp of $M_{Z F C}(T)$ and the field-dependent irreversibility between $M_{Z F C}$ and $M_{F C}$ reflecting a nonequilibrium character of thermodynamic behavior of the magnetic system studied are also characteristic of SG systems.

The low-field magnetization exhibits the magnetic hysteresis up to $200 \mathrm{~K}$. Figure 5 shows the hysteresis loops at 5 and $200 \mathrm{~K}$. The values of remanent magnetization and coercive force were estimated to be equal to $3 \cdot 10^{-3} \mathrm{emu} / \mathrm{g}$ and $60 \mathrm{Oe}$ at $5 \mathrm{~K}$ and $1.3 \cdot 10^{-3} \mathrm{emu} / \mathrm{g}$ and $30 \mathrm{Oe}$ at $200 \mathrm{~K}$, respectively. This fact can be also an evidence of ferro- 


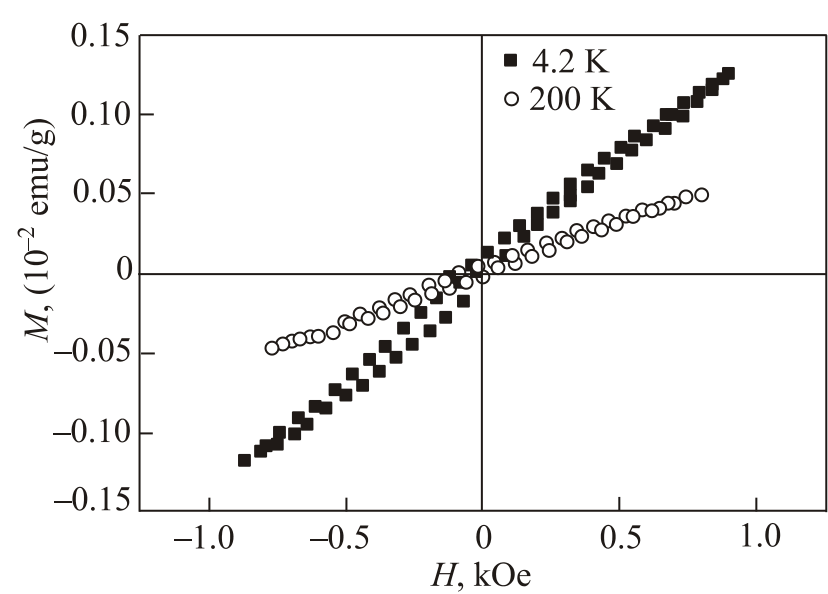

Fig. 5. Magnetic hysteresis loops at 4,2 and $200 \mathrm{~K}$.

magnetic interactions in this compound although a sign of the Weiss constant indicates the domination of antiferromagnetic exchange interactions. The presence of hysteresis and remanence over a wide temperature range is also a consequence of nonequilibrium state. The EPR spectra of the $\mathrm{Na}\left[\mathrm{FeO}_{6}\left(\mathrm{C}_{10} \mathrm{H}_{6} \mathrm{~N}\right)_{3}\right]$ complex show that the iron ions are randomly distributed in the organic matrix and their mutual interaction is rather weak [19].

In order to obtain additional information on magnetic behavior of the complex studied, the measurements of magnetization relaxation have been carried out. After ZFC process, the sample was kept in zero field for a waiting time $t_{w}$, before the magnetic field was applied. We have observed a relaxation of $M_{Z F C}(t)$ as a function of time. Inset to Fig. 4 shows the time evolution of $M_{Z F C}$ magnetization at 100 Oe after zero-field cooling from room temperature to $5 \mathrm{~K}$, for waiting time of $t_{w}=100 \mathrm{~s}$. The $M_{Z F C}$ versus $\log (t)$ plot has an inflection point at the time near the waiting time $t_{w}$. This $M_{Z F C}(t)$ behavior, called the aging effect, is also a characteristic feature of SG systems.

The difference between $M_{F C}$ and $M_{Z F C}$ below $T_{\text {ir }}$ and the presence of frequency-dependent peak on $\chi^{\prime}(T)$ dependence indicate the absence of magnetic long-range order in this complex.

The magnetization measurements results of the $\mathrm{Na}\left[\mathrm{FeO}_{6}\left(\mathrm{C}_{10} \mathrm{H}_{6} \mathrm{~N}\right)_{3}\right]$ complex performed at 1.5 and $5 \mathrm{~K}$ in magnetic field up to $90 \mathrm{kOe}$ are shown in Fig. 6. The behavior of the isotherms differs from the typical ferromagnetic behavior. The complete saturation is not attained even at $90 \mathrm{kOe}$ and $1.5 \mathrm{~K}$.

Thus, both types of magnetic measurements, i.e., ac susceptibility and dc magnetization, strongly support the hypothesis that a disordered spin state is realized in the $\mathrm{Na}\left[\mathrm{FeO}_{6}\left(\mathrm{C}_{10} \mathrm{H}_{6} \mathrm{~N}\right)_{3}\right]$ complex, namely, a spin-glass-like state with the freezing temperature $T_{f} \approx T_{\text {cusp }} \approx 17 \mathrm{~K}$. A spin-glass-like behavior manifests itself in several typical features, such as the cusp-like anomalies in both the ac susceptibility and the ZFC magnetization at $T_{f}$, frequency- and field- dependences of the $T_{\text {cusp }}$ temperature as well as

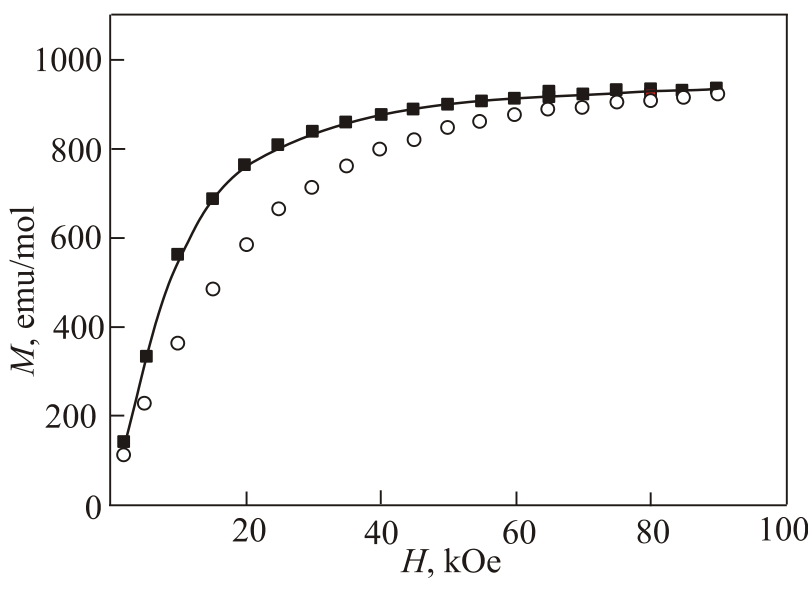

Fig. 6. Experimental magnetic field dependences of magnetization for the $\mathrm{Na}\left[\mathrm{FeO}_{6}\left(\mathrm{C}_{10} \mathrm{H}_{6} \mathrm{~N}\right)_{3}\right]$ complex at $1.5(\mathbf{\square})$ and $5 \mathrm{~K}(\mathrm{O})$. The Langevin function (Eq. (2)) was used to fit the $M(H)$ dependence to the experimental points at $1.5 \mathrm{~K}$ (solid line).

remanence and magnetic relaxation as a function of time. Experimental data obtained suggest that the transition from the paramagnetic to the spin-glass state, caused by freezing of decoupled paramagnetic spins, is more likely due to competing ferromagnetic and antiferromagnetic interactions.

However, the behavior of magnetization shows some deviations from typical SG, namely: i) irreversibility appears at $T \gg T_{f}$, ii) after field cooling, the magnetization decreases continuously with decreasing temperature, whereas in SG the magnetization is nearly constant at $T<T_{f}$. The latter fact can be understood if one assumes that at low temperatures a certain number of non-frozen moments still exists.

It should be also noted that the noticeable hysteresis, the existence of remanence and the dependence of $T_{\text {cusp }}$ on frequency may occur in other systems, such as superparamagnets.

Therefore, we have performed an analysis of revealed peculiarities of the magnetic characteristics of the $\mathrm{Na}\left[\mathrm{FeO}_{6}\left(\mathrm{C}_{10} \mathrm{H}_{6} \mathrm{~N}\right)_{3}\right]$ complex from the point of view of superparamagnetic behavior. We have supposed that the magnetic ions are coupled by AFM exchange interactions inside clusters $(\theta<0)$. The Langevin $I(H)$ function (Eq. (2)) was used to fit the $M(H)$ dependence to the experimental points. As it can be seen in Fig. 6, the fitting procedure leads to an acceptable agreement between the experimental curve and the theoretical dependence at $1.5 \mathrm{~K}$. This means that the superparamagnetic particles can be presented in the sample studied.

By fiting the theoretical Langevin function $I(H)$ :

$$
I(H)=n m\left[\operatorname{coth}\left(\frac{m H}{k_{B} T}\right)-\frac{k_{B} T}{m H}\right]
$$

where $m$ and $n$ are the magnetic moment of cluster and the number of clusters in mole of the compound, respectively, $k_{B}$ is the Boltzman constant, to the experimental magnetic 
field dependence of magnetization, $M_{\mathrm{ex}}(H)$, we have obtained the following values: $m / k=3.23 \cdot 10^{-4} \mathrm{emu} / \mathrm{mol}$ and $n m=986.8 \mathrm{emu} / \mathrm{mol}$. Then the magnetization value of one particle and the number of superparamagnetic particles in mole were found to be equal to $m=4.5 \cdot 10^{-20} \mathrm{emu} / \mathrm{mol}$ and $n=2.2 \cdot 10^{22} \mathrm{~mol}^{-1}$, respectively. Thus, the number of unit cells in one cluster, $N_{A} / n$ ( $N_{A}$ is the Avogadro number), is of about 27 , and the average size of a superparamagnetic particle is of about $16 \AA$ taking into account that the volume of unit cell is equal to $606 \AA^{3}$.The $\mathrm{x}$-ray diffraction data also show that the particles of the size of $100 \AA$ or more are not detected in the sample.

Using the blocking model of superparamagnetic particles we have also estimated the blocking $T_{f}$ temperature change as a function of frequency using the following expression [20]:

$$
f=f_{0} \exp \left(-E_{a} / k_{B} T\right),
$$

where $f_{0} \sim 10^{8}-10^{10} \mathrm{~s}^{-1}$ is the characteristic frequency for $\mathrm{SP}$ [21] and $E_{a}$ is the anisotropy energy of particles. Let us suppose that at experimental frequencies of $f=100 \mathrm{~Hz}$ and $20 f=2000 \mathrm{~Hz}$ the blocking temperatures are $T_{f 1}$ and $T_{f 2}$, respectively, which depend on the $E_{a}$ value. However, the relation between $T_{f 1}$ and $T_{f 2}$ does not depend on $E_{a}$ and has the distinct value at designated limits of relaxation $\left(f_{0}\right)$. Then the $T_{f 1} / T_{f 2}$ relation derived using the formula (3) has a form:

$$
T_{f 1} / T_{f 2}=\ln \left(20 f / f_{0}\right) / \ln \left(f / f_{0}\right),
$$

where $T_{f 1} / T_{f 2} \approx 0.8$. On other hand, the experiment gives the $T_{f 1} / T_{f 2}$ value of 0.87 , somewhat different from the theoretical value calculated.

In the spin-glass theory [22], the $T_{f 1} / T_{f 2}$ ratio is equal to one half of the square root from the right-hand part of the expression (4). This gives the value of 0.9 at $f_{0}=$ $=f_{S G} \sim 10^{11} \mathrm{~s}^{-1}\left(f_{S G}\right.$ is the characteristic frequency for $\mathrm{SG})$. So, in this case the agreement between experiment and theory is better.

From the experimental data it is evident that the iron-1-nitroso- $\beta$-naphthol complex has a very wide temperature interval, in which the relaxation phenomena are observed. This is in agreement with superparamagnetic properties of an assembly of stable single domain particles.

Thus, the experimental data obtained and their analysis strongly support the hypothesis of spin-glass-like behavior of the $\mathrm{Na}\left[\mathrm{FeO}_{6}\left(\mathrm{C}_{10} \mathrm{H}_{6} \mathrm{~N}\right)_{3}\right]$ complex. But there are some peculiarities of magnetic properties, which allow us to presume that this complex displays a superparamagnetic behavior at high temperatures and becomes spin-glass-like system with decreasing temperature.

\section{Conclusions}

In this work, we have presented a complete characterization of the magnetic behavior of the molecular-based magnet $\mathrm{Na}\left[\mathrm{FeO}_{6}\left(\mathrm{C}_{10} \mathrm{H}_{6} \mathrm{~N}\right)_{3}\right]$. The paramagnetic suscepti- bility data obey the Curie-Weiss law. Experimental data show the lack of long range magnetic order. The magnetization as a function of magnetic field does not reach a saturation up to $90 \mathrm{kOe}$ at $1.5 \mathrm{~K}$. On the basis of the experimental results and their analysis we have reported several typical evidences of a spin-glass-like properties of the $\mathrm{Na}\left[\mathrm{FeO}_{6}\left(\mathrm{C}_{10} \mathrm{H}_{6} \mathrm{~N}\right)_{3}\right]$ complex, resulting from the competition of ferromagnetic and antiferromagnetic interactions. The $\chi^{\prime}(T)$ dependence is accompanied by the onset of an out-of-phase component of the ac susceptibility. The compound studied shows a cusp-like anomalies in both the ac susceptibility and the ZFC magnetization near $T_{f}=17 \mathrm{~K}$, the frequency- and magnetic field- dependences of the $T_{\text {cusp }}$ temperature, the noticeable hysteresis and remanence as well as a time-dependent relaxation of $M_{Z F C}$ magnetization, which is expected on approaching the spin-glass state from the paramagnetic state.

However, some revealed peculiarities of magnetic behavior of the $\mathrm{Na}\left[\mathrm{FeO}_{6}\left(\mathrm{C}_{10} \mathrm{H}_{6} \mathrm{~N}\right)_{3}\right]$ complex are indicative of a possibility of an occurrence of superparamagnetic particles at high temperatures, e.g., the difference between $M_{F C}$ and $M_{Z F C}$ is much greater than expected in the SG phase at low temperatures; the irreversibility starts at $T \gg T_{f}$ indicating that a correlation between magnetic moments appears and decreases with increasing magnetic field, whereas in the SG case it occurs below $T_{f}$, the FC magnetization decreases monotonically with increasing temperature in contrast to spin glasses, where it is nearly constant at $T<T_{f}$.

Although superparamagnetic behavior should not be completely ruled out as an explanation of the freezing phenomena in studied sample, the preceding analysis strongly suggests that the spin-glass-like behavior is a more consistent explanation.

1. M. Deumal, J.M. Rawson, A.E. Goeta, J.A.K. Howard, R.C.B. Copley, M.A. Robb, and J.J. Novoa, Chem. Eur. J. 16, 2741 (2010).

2. R. Chiarelli, M.A. Novak, A. Rassat, and J.L. Tholence, Nature (London) 363, 147 (1993).

3. A. Rajca, J. Wongsrirtanakul, and S. Rajca, Science 294, 1503 (2001).

4. J.S. Miller, The Electrochemical Society Interface, Fall (2002).

5. W.A. Shelton, E. Apra, B.G. Sumpter, A. Saraiva-Souza, A.G. Souza Filho, J. Del Nero, and V. Meunier, Chem. Phys. Lett. 511, 294 (2011).

6. T. Makarova and F. Palacio, Carbon-Based Magnetism, Elsevier, Amsterdam (2006).

7. J.S. Miller and A.J. Epstein, Coordination Chem. Rev. 206, 651 (2000).

8. D. Gatteschi, R. Sessoli, and J. Villain. Molecular Nanomagnets, Oxford, University Press (2006).

9. J.M. Manriquez, G.T. Yee, R.S. Mclean, A.J. Epstein, and J.S. Miller, Science 252, 1415 (1991). 
10. M. Wan, C. Zhang, and C. Li, Solid State Commun. 87, 379 (1993).

11. P. Znou, S.M. Long, J.S. Miller, and A.J. Epstein, Phys. Lett. A 181, 71 (1993).

12. A.R. Harutyunyan, A.A. Kuznetsov, H. Szymczak, R. Szymczak, M. Baran, and A. Nabialek, J. Magn. Magn. Mater. 162, 338 (1996).

13. P. Zhou, B.G. Morin, J.S. Miller, and A.J. Epstein, Phys. Rev. B 48, 1325 (1993).

14. C.M. Wynn, M.A. Girtu, J. Zhang, J.S. Miller, and A.J. Epstein, Phys. Rev. 58, 8508 (1998).

15. S.J. Etzkorn, W. Hibbs, J.S. Miller, and A.J. Epstein, Polyhedron 22, 1751 (2003).

16. M.A. Gitu, J. Optoelectron. Adv. Mater. 4, 991 (2003).
17. E.I. Aksimentyeva, V.P. Dyakonov, V.N. Vasyukov, V.A. Shapovalov, and S. Piechota, Russ. J. Gen. Chem. 70, 823 (2000).

18. J.A. Mydosh, Spin Glasses: An Experimental Introduction, Taylor and Francis, London (1993).

19. V.V. Shapovalov, N.V. Vasyukov, V.A. Shapovalov, V.P. Dyakonov, O.I. Aksimentyeva, H. Szymczak, and S. Piechota, Molec. Cryst. Liq. Cryst. 384, 13 (2002).

20. T. Bitoh, K. Ohba, M. Takamatsu, T. Shirane, and S. Chikazawa, J. Magn. Magn. Matter. 154, 59 (1996).

21. L. Nèel, Compt. Rend. 228, 664 (1949).

22. A.P. Young, Phys. Rev. Lett. 50, 917 (1983). 